\title{
An Efficient Algorithm for Bifurcation Problems of Variational Inequalities
}

\author{
By H. D. Mittelmann*
}

\begin{abstract}
For a class of variational inequalities on a Hilbert space $H$ bifurcating solutions exist and may be characterized as critical points of a functional with respect to the intersection of the level surfaces of another functional and a closed convex subset $K$ of $H$. In a recent paper [13] we have used a gradient-projection type algorithm to obtain the solutions for discretizations of the variational inequalities. A related but Newton-based method is given here. Global and asymptotically quadratic convergence is proved. Numerical results show that it may be used very efficiently in following the bifurcating branches and that it compares favorably with several other algorithms. The method is also attractive for a class of nonlinear eigenvalue problems $(K=H)$ for which it reduces to a generalized Rayleigh-quotient iteration. So some results are included for the path following in turning-point problems.
\end{abstract}

1. Introduction. In the following we are concerned with the numerical computation of critical points of a functional $f: H \rightarrow \mathbf{R}, H$ a real Hilbert space, with respect to the intersection of a closed convex set $K \subset H$ and the level surfaces

$$
\partial S_{\rho}=\left\{u \in H, g(u)=\frac{1}{2} \rho^{2}\right\}
$$

of another (even) functional $g$. For theoretical results concerning existence, characterization of critical points, and relations to bifurcation theory we refer to the literature (see, for example, [1], [15], [19]). Under suitable assumptions a critical point $u_{0}$ satisfies the variational inequality

$$
\lambda_{0}\left(\nabla g\left(u_{0}\right), u-u_{0}\right) \geqslant\left(\nabla f\left(u_{0}\right), u-u_{0}\right) \quad \forall u \in K, \lambda_{0} \in \mathbf{R},
$$

and we are interested in the case $\lambda_{0}>0$.

Instead of treating the most general case we describe a class of such problems which is important in physical and mechanical applications. Some examples of this type will be considered later. $H$ will denote a function space of functions $u$ defined on a domain $\Omega \subset \mathbf{R}^{N}, N \geqslant 1$, and is usually a Sobolev space $H_{0}^{m}(\Omega)$, where only for simplicity the zero boundary conditions are included. The set $K$ will be either the whole space or a subset of the form

$$
K=\{u \in H, u \geqslant 0 \text { a.e. in } C, u \leqslant 0 \text { a.e. in } D\},
$$

where $C, D \subset \Omega$, so that $K$ is in fact a closed convex cone with vertex 0 .

While in the case $K=H$ several algorithms have been proposed for the determination of the critical points (see, for example, [9] and the papers cited there)

Received August 19.1981.

1980 Mathematics Subject Classification. Primary 65K10, 65N30, 65N25, 65L15, 73H05.

* This work was supported in part by the Deutsche Forschungsgemeinschaft and by the Department of Energy contract no. DOE-AS03-76-SF00326-PA \#30, while the author was a visitor at the Computer Science Department of Stanford University. 
and a vast literature deals with the corresponding differential equation problem, the theory for the case $K \neq H$ has only been developed recently (cf. [12] and the references in [13]). A numerical algorithm was given in [13]. Since this method as well as the algorithm to be presented below attack the discretized problem and have no simple analogue in the continuous case we shall restrict ourselves to finite-dimensional Hilbert spaces.

In [13] the problem of computing bifurcating solutions of variational inequalities was reduced to a standard optimization problem. A simple gradient-projection type method was used for its numerical solution. In Section 4 we describe a Newton-type method for the general problem considered in [13] and show in Section 6 that it may be used very efficiently for following the bifurcation branches for variational inequalities. Since the method is also attractive for the solution of a class of nonlinear eigenvalue problems, we formulate the method for the case $K=H$ first in the next section and present some numerical results in Section 5.

The contents of the following sections are

2. An algorithm for variational equalities.

3. Convergence proof.

4. An algorithm for variational inequalities.

5. Path following in turning-point problems.

6. Path following in bifurcation problems for variational inequalities.

2. An Algorithm for Variational Equalities. As indicated in the introduction, from now on we shall assume that the functionals $f$ and $g$ are either defined on a finite-dimensional Hilbert space $H$ or a problem of the class described above is discretized by, for example, a finite-difference or a finite element method yielding functionals $f_{h}, g_{h}$ defined on a space $H_{h}$, where $h$ denotes the discretization parameter. We shall assume that $H_{h}$ may be identified with Euclidean $n$-space, and we shall omit the subscript $h$.

In this and the following section we treat the case $K=H$ in which inequality (1.2) reduces to the variational equality.

$$
\lambda_{0}\left(\nabla g\left(u_{0}\right), u\right)=\left(\nabla f\left(u_{0}\right), u\right) \quad \forall u \in H .
$$

The original problem is the determination of critical points $u_{0}$ of the functional $f$ with respect to level sets (1.1) of the functional $g$.

We now make a few general assumptions on $f, g$, and we refer to the last sections where the examples show that the resulting class of problems covers interesting applications. Let the functional $f$ be twice Fréchet differentiable on $H$, and let $g$ be of the form

$$
g(x)=\frac{1}{2}(B x, x), \quad x \in H,
$$

where $B: H \rightarrow H$ is a linear, symmetric and positive definite operator. The elements of the finite-dimensional space $H$ will henceforth be denoted by $x, y$, etc.

Let there exist a constant $M=M(\rho)>0$ such that

$$
0<(\nabla f(x+y)-\nabla f(x), y) \leqslant M\|y\|^{2} \quad \forall x \in S_{\rho}, \forall y \in S_{2 \rho}, y \neq 0,
$$

and for simplicity let $M$ be chosen such that the following inequality also holds:

$$
(\nabla f(y), y) \leqslant M\|y\|^{2} \quad \forall y \in \partial S_{\rho} .
$$


Here we have used the notation $S_{\rho}=\left\{x \in H, g(x) \leqslant \frac{1}{2} \rho^{2}\right\}$. The norms used here and in the following are the Euclidean norm for $x \in H$ and the spectral norm for matrices $A \in L(H)$.

If $(2.2 \mathrm{a}),(2.2 \mathrm{~b})$ and

$$
f(0)=0, \quad \nabla f(0)=0
$$

are satisfied, then (2.1) always has the trivial solution, and it is well known that branches of solutions exist bifurcating from the eigenvalues of the linearized problem (cf. [12] and the references in [13]).

We now present an algorithm for the determination of local maxima of $f$ on the level surfaces (1.1) which is well defined under the assumptions of Theorem 2.10 below.

The algorithm for variational equalities. Let $x_{1} \in \partial S_{\rho}, \rho>0$, be arbitrary.

1. For $k=1,2, \ldots$ compute

$$
p_{k}=-H_{k} r_{k}
$$

where (formally) $H_{k}$ is the $n \times n$ principal submatrix of the inverse of

$$
D_{k}=\left[\begin{array}{cc}
F_{k}-\lambda_{k} B & -B x_{k} \\
-x_{k}^{T} B & 0
\end{array}\right],
$$

and we have used the notation $r_{k}=\nabla f\left(x_{k}\right), F_{k}=\nabla^{2} f\left(x_{k}\right), \lambda_{k}=r_{k}^{T} x_{k} / \rho^{2}$.

2. Determine a steplength $\alpha_{k}=2^{-j}$, where

$$
j=\min \left\{i \in N \cup\{0\}, f\left(x_{k}+2^{-i} p_{k}\right)-f\left(x_{k}\right) \geqslant 2^{-i-2} p_{k}^{T} r_{k}\right\} .
$$

3. Set

$$
x_{k+1}=\rho\left(x_{k}+\alpha_{k} p_{k}\right) /\left\|x_{k}+\alpha_{k} p_{k}\right\|_{B}
$$

where $\|\cdot\|_{B}=(,)_{B}^{1 / 2}$ and $(,)_{B}$ denotes the scalar product induced by $B$.

Remark 2.5. Algorithm (2.4) consists of a damped Newton step for the solution of the Kuhn-Tucker equations

$$
\nabla f(x)-\lambda B x=0, \quad-\frac{1}{2} x^{T} B x+\rho^{2} / 2=0,
$$

for updating $x_{k}$ starting from $x=x_{k}, \lambda=\lambda_{k}$ and a subsequent normalization to return to the level surface $\partial S_{\rho}$. The Langrange multiplier is updated by $\lambda_{k+1}=$ $r_{k+1}^{T} x_{k+1} / \rho^{2}$. Hence our method corresponds to the inverse iteration method with Rayleigh-quotient shift, while the Picard iteration considered in [6] corresponds to simple inverse iteration. For the matrix eigenvalue problem, i.e. $f(x)=\frac{1}{2}(A x, x), A$ symmetric, it is well known that the latter process exhibits linear convergence [22, $\mathrm{p}$. 619] while the first possesses locally cubic convergence properties ([22, p. 636], see also [18]). In the generalization to the nonlinear case considered here and in [6], the order stays the same for the ordinary inverse iteration while algorithm (2.4) will be shown to be quadratically convergent.

Remark 2.6. In order to show how a continuous analog of algorithm (2.4) would look, we derive it for the class of problems from [6]:

$$
\lambda L(u)=\varphi(x, u(x)), \quad x \in \Omega, \quad u(x)=0, \quad x \in \partial \Omega,
$$


where $L(u)=-\partial_{i}\left(a_{i k}(x) \partial_{k} u(x)\right)+a(x) u(x)$ with suitable assumptions on $a, a_{i k}, f$ (and using summation over repeated indices in the definition of $L$ ). We add the normalization

$$
(u, u):=\langle L(u), u\rangle=\rho^{2},
$$

where $\langle$,$\rangle denotes the L^{2}$-scalar product on $\Omega$. If now, for simplicity, we consider only the undamped case, a function $u_{k}$ satisfying $(2.7 \mathrm{~b})$ would be replaced by

$$
u_{k+1}=\rho\left(u_{k}+p_{k}\right) /\left\|u_{k}+p_{k}\right\|, \quad\|\cdot\|=(,)^{1 / 2},
$$

where $p_{k}$ is the first component of the solution $v=\left(v_{1}, v_{2}\right)$ of

$$
\begin{gathered}
\left(\varphi_{u}\left(x, u_{k}(x)\right)-\lambda_{k} L\right) v_{1}(x)-v_{2} L\left(u_{k}(x)\right)=-\varphi\left(x, u_{k}(x)\right), \quad x \in \Omega, \\
v_{1}(x)=0, \quad x \in \partial \Omega, \quad\left(u_{k}, v_{1}\right)=0, \quad \lambda_{k}=\left\langle\varphi\left(\cdot, u_{k}\right), u_{k}\right\rangle / \rho^{2},
\end{gathered}
$$

which may be obtained by determining $y_{k}, z_{k}$ from the two boundary value problems (2.8a) $\left(\varphi_{u}\left(x, u_{k}\right)-\lambda_{k} L\right) y_{k}(x)=-L\left(u_{k}(x)\right), \quad x \in \Omega, \quad y_{k}(x)=0, \quad x \in \partial \Omega$, (2.8b) $\left(\varphi_{u}\left(x, u_{k}\right)-\lambda_{k} L\right) z_{k}(x)=-\varphi\left(x, u_{k}(x)\right), \quad x \in \Omega, \quad z_{k}(x)=0, \quad x \in \partial \Omega$, and then setting

$$
p_{k}=z_{k}-\left(u_{k}, z_{k}\right)\left(u_{k}, y_{k}\right)^{-1} y_{k} .
$$

We observe, however, that the operator on the left-hand side of (2.8) becomes singular at a turning point and that Eq. (2.8a) cannot be satisfied there. Hence we are treating problem (2.7a) with a special form of the normalization as used in [8]. Then we apply a Newton step, however only for updating $u_{k}$. The normalization (2.7b) is responsible for some simplifications in (2.8), (2.9) compared with other choices.

We now state a local convergence theorem for algorithm (2.4). By $\{x\}^{\perp}$ we denote the orthogonal complement of $x \in H$ with respect to the scalar product $(,)_{B}$.

THEOREM 2.10. Let the assumptions (2.2) be satisfied for problem (2.1), and assume that $x_{0}$ is a solution of (2.1) for the parameter $\lambda_{0}$ and that $F\left(x_{0}\right)-\lambda_{0} B$ is negative definite on $\left\{x_{0}\right\}^{\perp}$. For $x_{1}$ sufficiently close to $x_{0}$ the sequence $\left\{x_{k}\right\}, k=1,2, \ldots$, generated by (2.4) converges to $x_{0}$ and. if $f \in C^{3}\left(U\left(x_{0}\right)\right)$, then the asymptotic $(Q-)$ order of convergence is two.

Remark 2.11. We have formulated this local theorem for the unrestricted case since the numerical applications we will treat in Sections 5 and 6 essentially need only this result. The theorem will be proved in the next section.

3. Convergence Proof. For the proof of Theorem 2.10 we need the following lemma. It suffices to prove it in the case $\rho=1$.

LEMMA 3.1. Under the assumptions of Theorem 2.10 let $U\left(x_{0}\right)$ be a neighborhood of $x_{0}$ such that for all $x \in U\left(x_{0}\right)$ and $\lambda(x)=\nabla f(x)^{T} x / \rho^{2}$

$$
y^{T}(F(x)-\lambda(x) B) y \leqslant-\beta\|y\|^{2}, \quad \beta>0, \forall y \in\{x\}^{\perp} .
$$

Let $x_{1} \in U\left(x_{0}\right)$ be chosen such that $\left\{x \in \partial S_{\rho}, f(x) \geqslant f\left(x_{1}\right)\right\} \subset U\left(x_{0}\right)$. If $x_{k} \in \partial S_{\rho}$ is generated by algorithm (2.4) with

$$
\alpha_{k}=\beta /(2 M \operatorname{cond}(B)),
$$


where $\operatorname{cond}(B)=\|B\|\left\|B^{-1}\right\|$, then $x_{k+1} \in \partial S_{\rho}$ and

$$
f\left(x_{k+1}\right)-f\left(x_{k}\right) \geqslant c\left\|p_{k}\right\|^{2}, \quad c>0 .
$$

Proof. Consider the case $\rho=1 . x_{k+1} \in \partial S_{\rho}$ is valid by construction of the algorithm. The following analysis is similar to that in the proof of Lemma 4.1 in [13] and is therefore given in concise form.

For a suitable $\tau \in(0,1)$ we have from $(2.2 b)$

$$
\begin{aligned}
& f\left(x_{k+1}\right)-f\left(x_{k}\right)=-\tau^{-1}(-\nabla f\left(x_{k}+\tau\left(x_{k+1}-x_{k}\right)\right) \\
&\left.\quad+\nabla f\left(x_{k}\right), \tau\left(x_{k+1}-x_{k}\right)\right)+\left(\nabla f\left(x_{k}\right), x_{k+1}-x_{k}\right) \\
& \geqslant-M\left\|x_{k+1}-x_{k}\right\|^{2}+\left(\nabla f\left(x_{k}\right), x_{k+1}-x_{k}\right) \\
& \geqslant 2 M\left\|B^{-1}\right\|\left(x_{k}+y_{k}, x_{k+1}-x_{k}\right)_{B}, \quad y_{k}=B^{-1} r_{k} /\left(2 M\left\|B^{-1}\right\|\right) .
\end{aligned}
$$

Hence

$$
f\left(x_{k+1}\right)-f\left(x_{k}\right) \geqslant d_{k}\left(x_{k}+y_{k}, x_{k}+\alpha_{k} p_{k}-\left\|x_{k}+\alpha_{k} p_{k}\right\|_{B} x_{k}\right)_{B},
$$

where $d_{k}=2 M\left\|B^{-1}\right\| /\left\|x_{k}+\alpha_{k} p_{k}\right\|_{B}>0$, and we show next that the second term on the right-hand side is nonnegative. This condition may be rewritten as

$$
\left(1-\left\|x_{k}+\alpha_{k} p_{k}\right\|_{B}\right)\left(1+\left(v_{h}, x_{h}\right)_{B}\right)+\alpha_{k}\left(v_{k}, p_{k}\right)_{B} \geqslant 0 .
$$

Writing the inverse of $D_{h}$ in $(2.4 \mathrm{~b})$ as

$$
D_{h}^{-1}=\left[\begin{array}{ll}
H_{k} & b_{k} \\
b_{h}^{T} & q_{k}
\end{array}\right],
$$

we deduce that

$$
\begin{gathered}
H_{k}\left(F_{k}-\lambda_{k} B\right)-b_{k} x_{k}^{T} B=E_{n} . \\
H_{k} B x_{k}=0,
\end{gathered}
$$

where $E_{n}$ is the identity matrix on $\mathbf{R}^{n}$. Hence $\left(x_{k}, p_{k}\right)_{B}=0$, and for $y=-H_{k} z$ we have $\left(y, x_{k}\right)_{B}=0$ and from (3.2), (3.8a) $\|y\|^{2} \leqslant \beta^{-1}(y, z)$. Applying this result for $z=r_{k}$, we derive

$$
\left\|p_{k}\right\|^{2} \leqslant \beta^{-1}\left(p_{k}, r_{k}\right)
$$

and $(3.8 \mathrm{~b})$ gives

$$
\left\|x_{k}+\alpha_{k} p_{k}\right\|_{B}^{2} \leqslant 1+\alpha_{k}^{2}\|B\|\left\|p_{k}\right\|^{2} .
$$

From (2.2c) we conclude that $\left(y_{k}, x_{k}\right)_{B} \leqslant \frac{1}{2}$. Hence

$$
\left(1+\left(y_{k}, x_{k}\right)_{B}\right)^{2} \leqslant 2\left(1+\left(y_{k}, x_{k}\right)_{B}\right)+\left\|p_{k}\right\|^{2} \beta^{2} /\left(4 M^{2}\left\|B^{-1}\right\|^{2}\|B\|\right),
$$

the last term being nonnegative, and thus

$$
\left(1+\alpha_{k}^{2}\left\|p_{k}\right\|^{2}\|B\|\right)\left(1+\left(y_{k}, x_{k}\right)_{B}\right)^{2} \leqslant\left(1+\left(y_{k}, x_{k}\right)_{B}+\beta \alpha_{k}\left\|p_{k}\right\|^{2} /\left(2 M\left\|B^{-1}\right\|\right)\right)^{2},
$$

from which now (3.6) immediately follows by taking square roots and using (3.9), (3.10).

Combining (3.5), (3.6), we obtain with $(2.2 \mathrm{~b})$ the inequalities

$$
f\left(x_{k+1}\right)-f\left(x_{k}\right) \geqslant\left(r_{k}, x_{k+1}-x_{k}\right) \geqslant M\left\|x_{k+1}-x_{k}\right\|^{2} \geqslant 0 .
$$


In order to show (3.4) we estimate, using (3.9), (3.10) and (2.2c),

$(3.12)\left(r_{k}, x_{k+1}-x_{k}\right)=\frac{\left(r_{k}, x_{k}\right)\left(1-\left\|x_{k}+\alpha_{k} p_{k}\right\|_{B}\right)+\alpha_{k}\left(r_{k}, p_{k}\right)}{\left\|x_{k}+\alpha_{k} p_{k}\right\|_{B}} \geqslant \frac{\alpha_{k} \beta\left\|p_{k}\right\|^{2}}{(2 L)}$,

where $L$ is an upper bound for $\left\|x_{k}+\alpha_{k} p_{k}\right\|_{B}$ on $U\left(x_{0}\right)$. The proof of the lemma is now complete.

In order to justify the choice of the Goldstein-Armijo stepsize rule instead of the constant $\alpha_{k}$ as in Lemma 3.1, we note that it may be shown as in [13] that

$$
\left\|x_{k+1}-\left(x_{k}+\alpha_{k} p_{k}\right)\right\|=O\left(\alpha_{k}^{2}\right),
$$

while (3.11), (3.12) yield an estimate linear in $\alpha_{k}$. The proof of the first part of Theorem 2.10 is now an immediate consequence of (3.4), (3.11).

It remains to show the asymptotically quadratic convergence. In $U\left(x_{0}\right)$ the matrix $D_{k}$ in (2.4b) is regular as a 'bordered' matrix. We next recall (cf. [6]) the expression for the derivative of an iteration function $\Phi$ as in (2.4).

The derivative of $\Phi(x)=y(x) /\|y(x)\|_{B}, y \in C^{1}$, is given by

$$
\Phi^{\prime}(x)=P_{y} y^{\prime}(x) /\|y(x)\|_{B},
$$

where $P_{z}=E_{n}-z z^{T} B /\|z\|_{B}^{2}$ is the orthogonal projector on $\{z\}^{\perp}$.

Now we show $\Phi^{\prime}\left(x_{0}\right) P_{x_{0}}=0$ from which the quadratic convergence follows using Lemma 10.1.7 in [16].

LEMMA 3.14. Under the assumptions of Theorem 2.10 the iteration function $\Phi$ of algorithm (2.4) satisfies

$$
\Phi^{\prime}\left(x_{0}\right) P_{x_{0}}=0
$$

Proof. We note that in (2.4c) $j=0$ will be chosen asymptotically and that then $\Phi(x)$ may be rewritten as (cf. (3.8b))

$$
\Phi(x)=\rho y(x) /\|y(x)\|_{B}, \quad y(x)=x-H(x)(\nabla f(x)-\lambda(x) B x) .
$$

The regularity of $D_{0}$. (3.8a) and Lemma 10.2.1 in [16] yield

$$
y^{\prime}\left(x_{0}\right)=E_{n}-H_{0}\left(F_{0}-\lambda_{0} B\right)=-b_{0} x_{0}^{T} B .
$$

$y\left(x_{0}\right)=x_{0}$ and (3.13) then finally give

$$
\Phi^{\prime}\left(x_{0}\right) P_{x_{0}}=-\rho P_{x_{0}} b_{0} x_{0}^{T} B P_{x_{0}}=0 .
$$

4. An Algorithm for Variational Inequalities. In this section we consider problem (1.2), (1.3). We present a globally convergent algorithm in the sense that it is not necessary, as in Theorem 2.10 , to choose $x_{1}$ in a sufficiently small neighborhood of a local maximum. Thus the following algorithm and theorem also generalize those of Section 2.

We look for local maxima of the functional $f$ defined on $H=\mathbf{R}^{n}$ over the set $K \cap \partial S_{\rho}, \partial S_{\rho}$ as in (1.1), with $g$ as in (2.2a) and $K$ a discrete analogue of (1.3):

$$
\begin{gathered}
K=\left\{x \in \mathbf{R}^{n}, x_{i} \geqslant 0, i \in J_{1}, x_{i} \leqslant 0, i \in J_{2}\right\}, \\
J_{1}, J_{2} \subset\{1, \ldots, n\}, \quad J_{1}=\left\{i_{1}, \ldots, i_{n_{1}}\right\}, \quad J_{2}=\left\{j_{1}, \ldots, j_{n_{2}}\right\} .
\end{gathered}
$$


We introduce some further notation (cf. [13]). Let $G=\left(g_{1}, \ldots, g_{n_{1}+n_{2}}\right)$, where $g_{k}=e_{i_{k}}, k=1, \ldots, n_{1}, g_{n_{1}+k}=e_{j_{k}}, k=1, \ldots, n_{2}, e_{i} \in \mathbf{R}^{n}$ the $i$ th unit vector. Then $K$ in (4.1) may be rewritten as

$$
K=\left\{x \in \mathbf{R}^{n}, G^{T} x \geqslant 0\right\} .
$$

For any $x \in \mathbf{R}^{n}$ let $I(x)=\left\{i \in\{1, \ldots, 2 n\}, g_{i}^{T} x=0\right\}$, and define $G_{I}=\left(g_{i}\right)_{i \in I}$, $Q_{I}=E_{n}-G_{I} G_{I}^{T}$. For $x=x_{k}$ denote $I_{k}=I\left(x_{k}\right), G_{k}=G_{I_{k}}$ and $Q_{k}$ analogously. We can now define

The algorithm for variational inequalities.

(4.3) Let $x_{1} \in K \cap \partial S_{\rho}$ be arbitrary. Set $k=1$ and $\mu_{k}=0, \mu_{k} \in\{0,1\}$.

1. Determine $I_{k}$ and $u_{k}=r_{k}-\lambda_{k} B x_{k}, \lambda_{k}=r_{k}^{T} x_{k} / \rho^{2}$. Terminate the iteration if $G_{k}^{T} u_{k} \leqslant 0$ and $\left\|Q_{k} u_{k}\right\|=0$.

2. Compute $\left|u_{k j}\right|=\max \left\{\left|u_{k i}\right|,\left(G_{k}^{T} u_{k}\right)_{i}>0\right\}$. If $\left\{\left(Q_{k} u_{k}, r_{k}\right)<\left|u_{k j}\right||| Q_{k} u_{k} \|\right.$ and $\left.\mu_{k}=0\right\}$ or $\left\|Q_{k} u_{k}\right\|=0$, then set $\tilde{I}_{k}=I_{k}-\{j\}$ and determine $\tilde{Q}_{k}$. Otherwise set $\tilde{I}_{k}=I_{k}, \tilde{Q}_{k}=Q_{k}$.

3. Replace $F_{k}-\lambda_{k} B$ in (2.4b) by $F_{k}-\lambda_{k} B-\tau_{k} E_{n}$, where $\tau_{k}=\max \left\{0, \delta+\sigma_{k}\right\}$ and $\sigma_{k}$ is the largest eigenvalue of $F_{k}-\lambda_{k} B$ on $\left\{x_{k}\right\}^{\perp} \cap\left\{x \in \mathbf{R}^{n}, \tilde{Q}_{k} x=x\right\}, \delta>0$ a given constant. Compute $p_{k}$ as the direction vector given by $(2.4 \mathrm{a})$ but in the variables $x_{k i}$ with $\left(\tilde{Q}_{k}\right)_{i i}=1$ (the free variables) only, fixing the others.

4. Determine the maximal admissible steplength $\bar{\alpha}_{k}$ and the steplength $\tilde{\alpha}_{k}$ as in (2.4), and set

$$
x_{k+1}=\rho\left(x_{k}+\alpha_{k} p_{k}\right) /\left\|x_{k}+\alpha_{k} p_{k}\right\|_{B},
$$

where $\alpha_{k}=\min \left\{\bar{\alpha}_{k}, \tilde{\alpha}_{k}\right\}$. If $\alpha_{k}=\bar{\alpha}_{k}$, then set $\mu_{k+1}=1$, otherwise $\mu_{k+1}=0$. Set $k=k+1$ and go to 1 .

THEOREM 4.4. Let the assumptions (2.2) be satisfied for problem (1.2). Assume that the set

$$
\Gamma=\left\{x^{*} \in K \cap \partial S_{\rho}, G^{* T} x^{*} \leqslant 0,\left\|Q^{*} x^{*}\right\|=0\right\}
$$

is finite and that $G^{* T} x^{*}<0$ for all $x^{*} \in \Gamma$ and $0<\delta<-\sigma^{*}(c f .3$ in (4.3)). Then the sequence $\left\{x_{k}\right\}, k=1,2, \ldots$, generated by algorithm (4.3) converges to a point $x^{*} \in \Gamma$. If $f \in C^{3}\left(U\left(x^{*}\right)\right)$, then the asymptotic $(Q-)$ order of convergence is two.

We first prove the analogue of Lemma 2.8 in the case $\rho=1$.

LEMMA 4.5. Let, under the assumptions of Theorem 4.4, $x_{k} \in K \cap \partial S_{\rho}$ be generated by algorithm (4.3) with steplength $\alpha_{k}=\delta /(2 M \operatorname{cond}(B))$. Then $x_{k+1} \in K \cap \partial S_{\rho}$ and

$$
f\left(x_{k+1}\right)-f\left(x_{k}\right) \geqslant \tilde{c}_{k}\left\{\begin{array}{l}
\left\|p_{k}\right\|^{2}, \quad \text { if } \mu_{k}=1, \\
\max \left\{\left\|p_{k}\right\|,\left|u_{k j}\right|\right\}^{2}, \quad \text { otherwise, }
\end{array}\right.
$$

where $\tilde{c}_{k}=c_{1}>0$ for $\mu_{k+1}=0$ and $\tilde{c}_{k}=c_{2} \bar{\alpha}_{k}, c_{2}>0$, for $\mu_{k+1}=1$.

Proof. The proof of (4.6) in the case $\mu_{k}=1$ follows closely the lines of the proof of Lemma 2.8. It is therefore not necessary here to give the details. We remark only 
that $p_{k}$ in (4.3) satisfies

$$
\left(x_{k}, p_{k}\right)_{B}=0, \quad \tilde{Q}_{k}\left(F_{k}-\lambda_{k} B-\tau_{k} E_{n}\right) \tilde{Q}_{k} p_{k}=-\tilde{Q}_{k} r_{k}
$$

and the analogue of (3.9) holds with $\beta$ replaced by $\delta$. Let now $\mu_{k}=0$ and $\tilde{I}_{k} \neq I_{k}$. Since the analogue to (3.8b) shows that $\tilde{Q}_{k} r_{k}$ in (4.7) may be replaced by $\tilde{Q}_{k} u_{k}$, which contains the component $u_{k j}$, the assumptions of Theorem 4.4 assure that for a positive constant $c\left\|p_{k}\right\| \geqslant\left\|\tilde{Q}_{k} u_{k}\right\| \geqslant\left|u_{k j}\right|$. This proves (4.6) for $\tilde{I}_{k} \neq I_{k}$. If $\tilde{I}_{k}=I_{k}$, then the strategy in 2. of (4.3) guarantees that $\left\|Q_{k} r_{k}\right\| \geqslant\left|u_{k j}\right|$ while (4.7) gives $c\left\|p_{k}\right\| \geqslant\left\|Q_{k} r_{k}\right\|$. This completes the proof of the lemma.

The proof of the first part of Theorem 4.4 need also not be given in detail here since it follows from combining the arguments of the proofs of Theorem 2.10 above and Theorem 3.1 in [13]. This shows that, for all sufficiently large $k, I_{k}=I\left(x^{*}\right)$, $x^{*} \in \Gamma$ and $\tau_{k}=0$. Thus the steplength $\alpha_{k}$ will finally be chosen equal to 1 , and the asymptotically quadratic convergence then follows as in the proof of Theorem 2.10.

Remark 4.8. For a practical application of algorithm (4.3) a way of choosing the regularization parameter $\tau_{k}$ has to be given. For a more general class of optimization problems a procedure for this purpose is described in [20].

5. Path Following in Turning-Point Problems. In this section we consider the same class of problems as in [6], namely the nonlinear eigenvalue problem (cf. (2.7a))

$$
\lambda L(u)=\varphi(x, u(x)), \quad x \in \Omega, \quad u(x)=0, \quad x \in \partial \Omega,
$$

where $\lambda \in \mathbf{R}, \lambda>0$, and $L$ is a uniformly elliptic formally selfadjoint differential operator on the bounded domain $\Omega \subset \mathbf{R}^{n}$. Generalizations, for example, to higher order differential operators or other boundary conditions are possible. Conditions (2.2) have to be satisfied in the continuous case and for the discretization. We shall restrict ourselves to the example (cf. e.g. [4])

$$
L(u)=-\Delta u, \quad \varphi(x, u)=\exp (u /(1+\varepsilon u)), \quad \varepsilon \geqslant 0,
$$

and $N=2$. For $\varepsilon=0$ (5.1), (5.2) is usually called Bratu's problem.

There has been a great interest in the numerical solution of similar problems, see, for example, the papers mentioned in Section 5.6 of [14]. For theoretical results on problems of the type (5.1) see, for example, [5], [7], [19]. It is well known that (5.1), (5.2) has a solution diagram as shown in Figure 1 in dimensions $N=1,2$. The points marked in the figure represent, for $\varepsilon<\varepsilon^{*}$, one or two (quadratic) simple turning points and, for $\varepsilon=\varepsilon^{*}$, a nonsimple turning point.

The problem of following the solution branch and also the problem of determining the simple respectively the nonsimple turning points numerically presents in principle no difficulties (cf. [2], [14], [21]). However, using e.g. Keller's pseudoarclength-continuation technique the stepsize has to be suitably controlled near the limit point, and the question of efficiency arises in particular if the linear systems are solved by elimination methods. In [2] a multigrid (MG)-method was suggested for the approximate solution of (5.1), (5.2). The pseudo-arclength normalization was added (cf. [8]), and the resulting system was solved by block-elimination as utilized also in Remark 2.6. Hence a differential operator was discretized, which becomes singular in the turning point. The corresponding singularity of the discrete operator 
on one of the grids used in the MG-method made it necessary to modify this algorithm considerably in order to be able to pass the limit point. These modifications may not have been necessary, if instead the inflated system would have been treated directly. The resulting system has a regular matrix in the neighborhood of solutions. However, the matrix is not definite on the whole space, so that is is open how the MG-method would perform. This question will be investigated in the future. For an application of MG using Rayleigh-quotient iteration to the linear eigenvalue problem cf. [11].

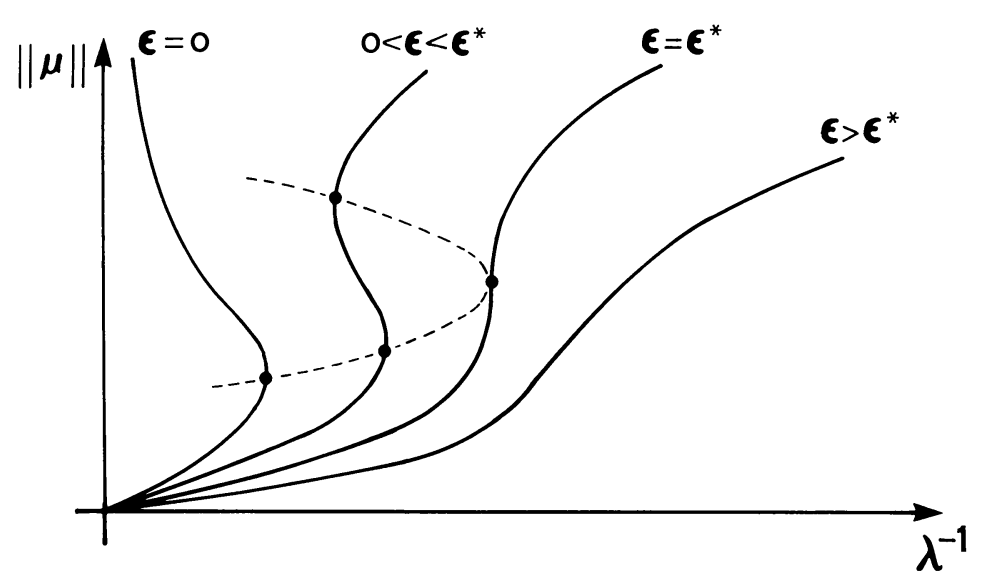

FIGURE 1

Solution diagram for problem (5.1), (5.2) for different vaiues of $\varepsilon$

It is a well-known procedure to use a norm of $u$ as a continuation parameter, and a numerical method for this is, for example, the Picard iteration of [6]. The algorithms of Sections 2 and 4 can be used analogously. They have the advantage of quadratic convergence, while Fast-Poisson-Solvers in the special case $L=-\Delta$ could in general not be utilized. It should, however, as pointed out above, be possible to use MG-algorithms.

We compare now algorithm (2.4) and that of [6] on the above problem. Since it is not our aim to compute the solution to a high accuracy, we have chosen a low order finite element method on a relatively coarse mesh. Problem (5.1), (5.2) may be written in the variational form

$$
\begin{gathered}
\lambda(\nabla g(u), v)=(\nabla f(u), v) \quad \forall v \in H_{0}^{1}(\Omega), \\
g(u)=\frac{1}{2} \int_{\Omega}\left(u_{x}^{2}+u_{y}^{2}\right) d x d y, \quad f(u)=\int_{\Omega} \exp (u /(1+\varepsilon u)) d x d y .
\end{gathered}
$$

$\Omega$ was taken as the unit square and linear finite elements were used on the standard triangulation obtained from a square mesh with meshwidth $h . f$ was evaluated by numerical integration with weights $h^{2} / 6$ and the midpoints of the edges of a triangle as integration points. This gave rise to the usual five-point difference matrix $B$ and a seven-band matrix $A$. Table 1 shows the results for two values of $\varepsilon<\varepsilon^{*}$. 
TABLE 1

Computed points on the solution branch for problem (5.1), (5.2) near the turning points and necessary number of iterations for different algorithms.

$\begin{array}{ccccc}\varepsilon & \rho & \lambda^{-1} & \operatorname{Alg}(2.4) & \text { Picard } \\ 0.0 & 30 & 6.712380 & 4(4) & 9(17) \\ 0.0 & 36 & 6.910483 & 2(3) & 8(16) \\ 0.0 & 42 & 6.882701 & 2(3) & 8(16) \\ 0.0 & 48 & 6.681038 & 2(3) & 9(16) \\ 0.2 & 72 & 9.278187 & 3(3) & 10(18) \\ 0.2 & 80 & 9.291875 & 2(3) & 9(18) \\ 0.2 & 88 & 9.265477 & 2(3) & 9(18) \\ 0.2 & 96 & 9.211836 & 2(3) & 8(18) \\ 0.2 & 360 & 7.341984 & 2(3) & 9(20) \\ 0.2 & 440 & 7.237885 & 2(3) & 9(20) \\ 0.2 & 520 & 7.230358 & 2(3) & 9(20) \\ 0.2 & 600 & 7.285922 & 2(3) & 8(20)\end{array}$

For either method the number of iterations is given required to compute the solution to about eight decimal places with the number of iterations for maximal accuracy (double precision FORTRAN on an IBM 370-168) given in parentheses. The starting vector for $\rho=30$ and for both algorithms was $x_{0}=e /\|e\|_{B}, e=$ $(1, \ldots, 1)^{T} \in \mathbf{R}^{n}, n=((1-h) / h)^{2}, h=1 / 12$. The approximate solution for each $\rho$-value was then, after normalization, used as starting guess for the next $\rho(\varepsilon)$-value. Algoritm (2.4) could in each case be used with $\alpha_{k}=1$. The linear system for the symmetric but in general indefinite matrix $D_{k}$ in $(2.4 \mathrm{~b})$ may be solved, for example, by any conjugate gradient method applicable to such problems (see, for example, [3]), and even special elimination procedures are easy to derive. We used algorithm SYMMLQ [17] which without any scaling or preconditioning needed about 35 iterations to solve the system in each step.

The iterates of our algorithm converged quadratically from the beginning. The steps in $\rho$ for (2.4) could be chosen large as the results show, but not arbitrarily large, while the Picard iteration did not seem to have similar restrictions. So an alternative to damping in (2.4) could be to first execute some Picard steps and then to use algorithm (2.4) with stepsize 1.

In this section we have seen that algorithm (2.4) may be used very efficiently in the following of solution branches for problems of the type (5.1). For more general bifurcation problems a natural procedure would be to use alternately continuation with respect to $\lambda$ or to the norm of $x$ (cf. Section 6) switching when the steplength in one of the methods has to be chosen below a suitable tolerance. The use of MG-methods may be possible, however, conjugate gradient algorithms provide an efficient and generally applicable procedure for the solution of the linear systems.

6. Path Following in Bifurcation Problems for Variational Inequalities. In this section we again restrict the numerical computations to a simple but illustrative example. We apply algorithm (4.3) to the discretization used in [13] of the buckling 
problem for an axially compressed beam with lateral supports. The variational inequality is

$$
\begin{gathered}
\lambda\left(\nabla g\left(u_{0}\right), u-u_{0}\right) \geqslant\left(\nabla f\left(u_{0}\right), u-u_{0}\right) \quad \forall u \in K, \\
f(u)=\int_{0}^{1}\left[\left(1+u^{\prime 2}\right)^{1 / 2}-1\right] d x, \quad g(u)=\frac{1}{2} \int_{0}^{1} u^{\prime \prime 2} d x, \\
K=\left\{u \in H_{0}^{2}[0,1], u(C) \geqslant 0, u(D) \leqslant 0\right\} .
\end{gathered}
$$

Hermite cubic finite elements on an equidistant grid of width $h$ and suitable numerical integration are used yielding the discrete functionals $f_{h}, g_{h}$ (cf. [13]). Of physical interest are the solutions $u_{h}$ branching from the trivial solution at the largest eigenvalue $\lambda_{h 1}$ with eigenvector $u_{h 1}$ of the linearized problem.

To our knowledge no reasonably efficient algorithms are available which are globally convergent to $u_{h 1}$ if $K \neq H$, except in special cases (see, for example, Corollary 4.2 in [13]). In [10] a constructive existence proof for the restricted solutions has been given, in which they are obtained as bifurcating solutions of a penalized version of the unrestricted problem $(K=H)$. In that paper, however, only eigenfunctions can be determined corresponding to eigenvalues which are smaller than the largest eigenvalue of the unrestricted problem for which the corresponding eigenfunction with suitably chosen sign is in the interior of $K$. Hence the physically interesting case is excluded.

We assume now that $\left(\lambda_{h 1}, u_{h 1}\right)$ and the corresponding set of active constraints are known and try to follow the branch bifurcating from $\left(\lambda_{h 1}, 0\right)$. In [13] it was suggested that augmented Lagrangian methods could advantageously be used for this purpose. The following results, however, show that algorithm (4.3), which here essentially reduces to (2.4) in the subspace of the free variables, is the most efficient method among several algorithms. We compared it with SALMNA, an augmented Lagrangian-type algorithm using Newton's method from the NPL-library and also part of the NAG-library. Another natural candidate for a comparison is $\lambda$-continuation (see, for example, [8]) which in this case should not be inferior to pseudoarclength-continuation:

Let $\left(u^{0}, \lambda^{0}\right)$ on the branch be given. Compute $u_{\lambda}\left(u^{0}, \lambda^{0}\right)$ from

$$
\left(F\left(u^{0}\right)-\lambda^{0} B\right) u_{\lambda}=B u^{0} \text {. }
$$

Then set $u_{0}=u^{0}+\left(\lambda-\lambda^{0}\right) u_{\lambda}$ and for $k=0,1, \ldots$ iterate according to

$$
\left(F\left(u_{k}\right)-\lambda B\right)\left(u_{k+1}-u_{k}\right)=-\nabla f\left(u_{k}\right)+\lambda B u_{k} .
$$

Hence after an Euler predictor step several Newton steps are executed to compute the solution for the given $\lambda$. Finally, Picard iteration is applied here, too.

We have restricted the computations to the problem (6.1) with $C=\{1 / 3\}$, $D=\{2 / 3\}$. Largest eigenvalue and corresponding eigenfunctions for this case, there are two symmetric eigenfunctions, have been computed analytically in [13]. Table 2 shows some typical results for $h=1 / 24$. Again the number of iterations is given required to compute the solution to eight decimal places, respectively, to the maximal attainable accuracy. For SALMNA the numbers represent for the latter case only the number of second order derivative (function value and first order derivative) evaluations. 
TABLE 2

Computed points on the bifurcating branch for problem (6.1)

and iteration counts for different algorithms.

$\begin{array}{rccccc}\rho & \lambda_{h \rho} & \text { Alg. (4.3) } & \text { Picard } & \lambda \text {-cont. } & \text { SALMNA } \\ 1 & .144644 \mathrm{E}-1 & 2(3) & 14(28) & 5(6) & 2(9) \\ 2 & .139243 \mathrm{E}-1 & 2(3) & 15(28) & 4(5) & 2(8) \\ 10 & .799582 \mathrm{E}-2 & 3(4) & 16(30) & 5(6) & 9(14) \\ 100 & .103968 \mathrm{E}-2 & 3(4) & 14(28) & 7(8) & 70(104)\end{array}$

For each algorithm the normalized eigenfunction of the linear eigenvalue problem was used as starting solution for $\rho=1$, and the corresponding Rayleigh-quotient was used as starting value for the Lagrange multiplier in SALMNA. Then the solutions on the branch for the given sequence of $\rho$-values were computed by continuing analogously to $\rho=2,10,100$. The corresponding $\lambda$-values were used as the sequence for the $\lambda$-continuation.

The results show that our method is also very efficient for following bifurcating branches of variational inequalities. The behavior of the Picard iteration is similar to that in Section 5, while for $\lambda$-continuation the convergence of the Newton iterates was not quadratic from the start, which resulted in considerably more iterations especially for larger $\rho$-steps. The iteration counts for this method in Table 2 do not include the predictor step. Finally, the performance of the general purpose routine SALMNA suggests that augmented Lagrangian methods are not able to compete with algorithm (4.3) for the special class of optimization problems considered here. By modifying the subroutine suitably it should, however, be possible to reduce the extremely high expense needed for larger $\rho$-steps.

For the solution of the linear systems again SYMMLQ was used, which even after a scaling of the sytem needed more than $n$ iterations. The number of iterations, however, was only slightly larger than for the solution of the system in the Picard iteration, which has a definite matrix. So this difficulty is caused by the unfavorable eigenvalue distribution for this fourth-order problem and, if conjugate gradient methods are to be used for the linear systems, a suitable preconditioning should be chosen to further reduce the necessary work.

Abteilung Mathematik

Universität Dortmund

Postfach 500500

D-4600 Dortmund

Federal Republic of Germany

1. M. S. Berger, "A bifurcation theory for nonlinear elliptic partial differntial equations and related systems," Bifurcation Theory and Nonlinear Eigenvalue Problems (J. B. Keller and S. Antman, eds.), Benjamin, New York, 1969.

2. T. F. C. Chan \& H. B. Keller, "Arclength continuation and multi-grid techniques for nonlinear elliptic eigenvalue problems,” SIAM J. Sci. Statist. Comput., v. 3, 1982, pp. 173-194.

3. R. Chandra, Conjugate Gradient Methods for Partial Differential Equations, Techn. Rep. no. 129, Dept. of Comp. Sci., Yale University, 1978.

4. L. J. Fradkin \& G. C. WAKE, "The critical explosion parameter in the theory of thermal ignition," J. Inst. Math. Appl., v. 20, 1977, pp. 471-484. 
5. I. M. Gelfand, "Some problems in the theory of quasilinear equations," Amer. Math. Soc. Transl., v. 29. 1963, pp. 295-381.

6. K. GEORG, "On the convergence of an inverse iteration method for nonlinear elliptic eigenvalue problems," Numer. Math., v. 32, 1979. pp. 69-74.

7. H. B. Keller, "Some positone problems suggested by nonlinear heat generation," in Bifurcation Theory and Nonlinear Eigenvalue Problems (J. B. Keller and S. Antman, eds.). Benjamin. New York. 1969.

8. H. B. Keller, "Numerical solution of bifurcation and nonlinear eigenvlaue problems," in Applications of Bifurcation Theory (P. H. Rabinowitz, ed.), Academic Press, New York, 1977.

9. A. Kratochvil \& J. Necas, "Gradient methods for the construction of Ljusternik-Schnirelmann critical values," RAIRO Anal. Numerr., v. 14, 1980, pp. 43-54.

10. M. KUCERA, "A new method for obtaining eigenvalues of variational inequalities based on bifurcation theory," Časopis Pěst. Mat., v. 104. 1979. pp. 389-411.

11. S. F. McCormick, "A mesh refinement method for $A x=\lambda B x$, Math. Comp.. v. 36, 1981, pp. 485-498.

12. E. MiersemanN, "Verzweigungsprobleme für Variationsungleichungen.” Math. Nachr., v. 65, 1975. pp. 187-209.

13. H. D. MittelmanN, "Bifurcation problems for discrete variational inequalities," Math. Methods Appl. Sci., v. 4. 1982, pp. 243-258.

14. H. D. Mittelmann \& H. Weber, "Numerical methods for bifurcation problems-A survey and classification," in Bifurcation Problems and Their Numerical Solution (H. D. Mittelmann and H. Weber, eds.). ISNM 54, Birkhäuser-Verlag. Basel, 1980.

15. J. NeCAS, "Approximation methods for finding critical points of even functionals," Trudy Matem. Inst. A.N. SSSR, v. 134, 1975, 235-239.

16. J. M. ORTEGA \& W. C. Rheinboldt, Iterative Solution of Nonlinear Equations in Several Variables, Academic Press, New York and London, 1970.

17. C. C. Paige \& M. A. Saunders, "Solution of sparse indefinite systems of linear equations," SIAM J. Numer. Anal., v. 12, 1975, pp. 617-629.

18. G. Peters \& J. H. Wilkinson, "Inverse iteration, ill-conditioned equations and Newton's method," SIAM Rev., v. 21, 1979, pp. 339-360.

19. P. H. Rabinowitz, "Variational methods for nonlinear elliptic eigenvalue problems," Indiana Univ. Math. J., v. 23, 1974, pp. 729-754.

20. P. SPElluCCI, "Some convergence results for generalized gradient projection methods," Methods of Operations Research, Vol. 36, Verlag Anton Hain. Königsstein, 1980, pp. 271-280.

21. A. Spence \& B. Werner, "Non simple turning points and cusps," IMA J. Numer. Anal. (To appear.)

22. J. H. Wilkinson, The Algehraic Eigenvalue Problem, Clarendon Press, Oxford, 1965. 